\title{
Use of parathyroid hormone analog (Teriparatide) in patients with chronic hypoparathyroidism after total thyroidectomy: a case report
}

\author{
${ }^{1}$ Rai A, ${ }^{2}$ Karki P, ${ }^{3}$ Paudel D, ${ }^{4}$ Maskey R \\ ${ }^{1}$ Senior resident, ${ }^{4}$ Additonal Professor, Department of Internal Medicine, BPKIHS, Dharan, Nepal, ${ }^{2}$ Senior \\ Resident, ${ }^{3}$ Assistant Professor, Department of ENT, BPKIHS, Dharan, Nepal
}

\begin{abstract}
Background: Hypoparathyroidism and hypocalcemia is a common postoperative complication, after total thyroidectomy due to thyroid cancer. Standard treatment with supplementation of calcium and vitamin $\mathrm{D}$ analogs, usually treat this condition. In some patients, hypoparathyroidism is refractory to standard treatment plus intermittent calcium infusions with persistent low serum calcium levels and associated clinical complications. Attempts have been made to add recombinant human parathormone (rhPTH) to the treatment schedule. To our knowledge, this is the first time that we encounter a patient suffering from treatment-refractory postsurgical hypoparathyroidism who was treated with teriparatide. Case presentation: Male (31 years) with postoperative hypoparathyroidism, after total thyroidectomy due to papillary thyroid cancer, several weeks after the surgery still required intermittent intravenous calcium infusions because of tetany symptoms. He had persistent hypocalcemia despite oral treatment with up to $1 \mathrm{ug}$ calcitriol and $4 \mathrm{~g}$ calcium per day necessitating additional intravenous administration of calcium gluconate intermittently. This time, Teriparatide treatment was introduced at once daily 50 micrograms (mcg) subcutaneous injection, while doses of calcium and calcitriol were gradually decreased depending on the response of serum total and ionized calcium taken periodically, which resulted in total resolution of hypocalcemia symptoms and the achievement and maintenance of laboratory normocalcaemia in just 5 days. Conclusion: Treatment refractory chronic hypoparathyroidism may be seen in some cases after total thyroidectomy. Furthermore, the use of recombinant human parathyroid hormone analog (Teriparatide) allows for the control of recurrent hypocalcemia reducing the daily dosage of calcium and vitamin D. Finally, regular intravenous calcium administration was no more needed.
\end{abstract}

Key words: Postoperative Chronic Hypoparathyroidism, Recurrent Hypocalcemia, Teriparatide

\section{Introduction}

Hypoparathyroidism is a common complication following bilateral thyroid surgery. Postthyroidectomy hypoparathyroidism is due to inadvertent excision, damage, or devascularization of the parathyroid gland(s). Hypocalcemia, in most cases, is transient, which usually resolves after 2-3 months with oral calcium and vitamin D supplementation alone. Meanwhile, $0.12 \%-4.6$ $\%$ of cases may have chronic hypoparathyroidism

Correspondence Author

Dr. Avinash Rai, Senior Resident Department of Internal Medicine, B.P. Koirala Institute of Health Sciences, Dharan, Nepal extending beyond 6 months ${ }^{1}$.

Hypoparathyroidism is characterized by low parathyroid hormone (PTH) and low calcium levels. The main clinical features of the disease are hypocalcemic symptoms such as perioral numbness, limb paresthesia, and carpopedal muscle spasms. Acute hypocalcemia can be a medical emergency with severe and potentially life-threatening complications such as laryngeal spasms, tetany, and seizures that may occur requiring intravenous calcium ions. Supplementation of calcium, active vitamin $\mathrm{D}$, and at times, thiazide diuretics may be sufficient for most of the individuals with iatrogenic hypoparathyroidism. Despite the valiant efforts, some cases may be difficult to control 
with conventional therapy, even after high doses are employed. Moreover, prolonged use of high doses can cause hypercalciuria, nephrocalcinosis, ectopic soft tissue calcification, and subsequent renal impairment ${ }^{2}$. besides, conventional therapy does not alleviate Quality of life complaints, nor does it reverse bone remodeling abnormalities of the disease $\mathrm{e}^{3}$. To sum things up, conventional therapy does not provide benefits that a hormone replacement therapy provides for the lack of PTH in chronic hypoparathyroidism.

Hypoparathyroidism is the last remaining classic endocrine deficiency disorder for which hormone replacement therapy was not approved until recently. Over the past decade, the study of full-length natural PTH (1-84) and short active N-terminal PTH (teriparatide (PTH 1-34)) have introduced a new era of management of this disease. In 2015, the United States Food and Drug Administration (FDA) approved the use of recombinant human PTH (1-84) in treating patients with classical treatment-resistant hypoparathyroidism ${ }^{4}$. Regarding our clinical settings, the incidence of chronic hypoparathyroidism post-thyroid surgery is rare. Furthermore, the usage of Teriparatide (PTH 1-34) as hormone replacement therapy in such a case is itself a first of its kind here.

\section{Case Report}

A 31-year-old male, non-smoker, non-alcohol consumer, presented with bilateral upper/lower limb paresthesia and carpopedal spasm. He does not have any history of hypertension, diabetes mellitus, tuberculosis. His family history was not significant. The patient had undergone total thyroidectomy due to papillary carcinoma thyroid 8 months back. Thereafter, the patient developed postoperative hypoparathyroidism and was under calcium $3 \mathrm{~g} /$ day and active vitamin D (calcitriol) $0.25 \mathrm{ug} /$ day for hypocalcemia. The patient had been on intermittent calcium gluconate injections for recurrent tetany symptoms on an emergency basis.

This time (2020/07/08), he was admitted to the department of Internal medicine with the chief complaint of bilateral upper and lower limb tingling sensation, weakness, myalgia, and severe spasmodic pain. At the time of presentation, his physical examination results were normal, except for thyroidectomy scars and carpopedal spasm (see figure 1).

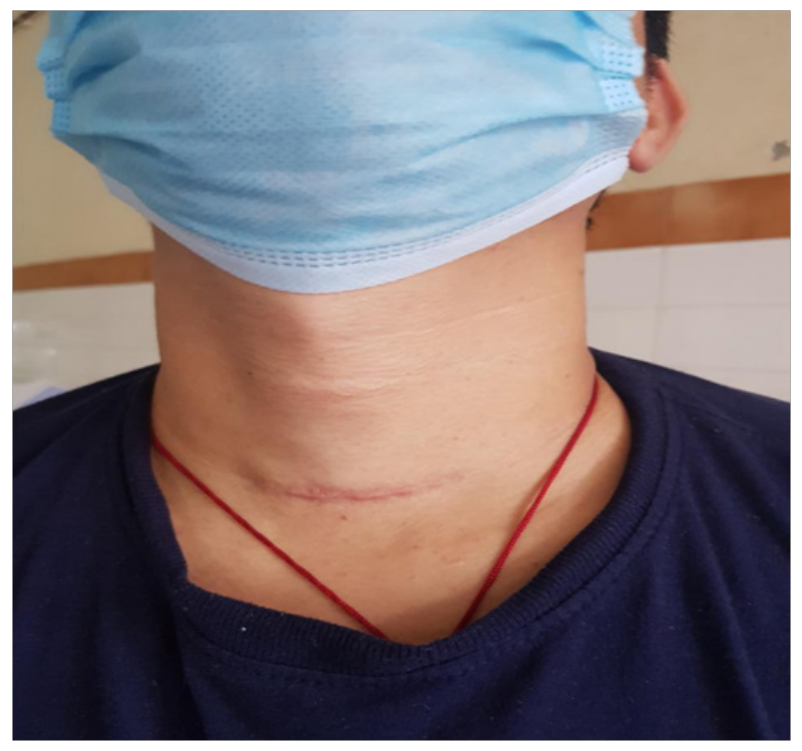

Figure 1: 31 year old male with status post total thyroidectomy, with thyroidectomy scar.

On examination, chvostek's sign and trousseau's sign were positively elicited. His vitals were: Pulse rate 90 bpm, Blood Pressure 120/70 mmHg, Respiratory rate 18/min, Temp 37.3 C.

The laboratory values of the patient after hospital admission are shown in table 1. 
Table 1: Clinical parameters of the patient during time of admission.

\begin{tabular}{|l|l|l|l|}
\hline Clinical parameter & Value (normal range) & Clinical parameter & Value (normal range) \\
\hline Total calcium & $5.5 \mathrm{mg} / \mathrm{dl}(8.4-10.2)$ & Hemoglobin & $13.0 \mathrm{gm} / \mathrm{dl}(11-16)$ \\
\hline Ionised calcium & $0.76 \mathrm{mmol} / \mathrm{L}(1.05-1.27)$ & RBS & $94 \mathrm{mg} / \mathrm{dl}(<140)$ \\
\hline Phosphorus & $3.6 \mathrm{mg} / \mathrm{dl}(2.5-4.5)$ & Urea & $21 \mathrm{mg} / \mathrm{dl}(10-50)$ \\
\hline Magnesium & $0.81 \mathrm{mmol} / \mathrm{L}(0.66-1.07)$ & Creatinine & $1.0 \mathrm{mg} / \mathrm{dl}(0.6-1.3)$ \\
\hline Albumin & $4.7 \mathrm{~g} / \mathrm{dL}(3.5-5)$ & ALP & $26 \mathrm{U} / \mathrm{L}(35-130)$ \\
\hline TSH & $0.001 \mathrm{uIU} / \mathrm{ml}(0.3-4.5)$ & Sodium & $144 \mathrm{mmol} / \mathrm{L}(135-145)$ \\
\hline PTH & $2.426 \mathrm{pg} / \mathrm{mL}(6-80)$ & Potassium & $4.6 \mathrm{mmol} / \mathrm{L}(3.5-5.0)$ \\
\hline Vitamin D & $64.7 \mathrm{ng} / \mathrm{mL}(30-80)$ & & \\
\hline
\end{tabular}

Abbreviations: TSH - Thyroid stimulating hormone; PTH - Parathyroid hormone; RBS - Random blood sugar; ALP - Alkaline phosphatase.

ECG showed a normal sinus rhythm. Intravenous calcium gluconate was given at bolus dose and continued on infusion. His oral medication dose was increased to calcitriol $1 \mathrm{ug} /$ day and elemental calcium $4 \mathrm{~g}$ /day. On intravenous calcium infusions, Serum calcium level returned to normal range, but after stopping calcium infusion hypocalcemia recurred. As hypomagnesemia is associated with hypocalcemia, correction of magnesium deficiency was done by intravenous magnesium infusion. We could not maintain normocalcemia even after continuous infusions of calcium gluconate. Symptoms of peri-oral tingling sensation, carpopedal spasm would not completely recover. We decided to start a parathyroid hormone analog (teriparatide) replacement therapy on this patient. We initiated a once daily $50 \mathrm{mcg}$ subcutaneous injection of recombinant human PTH (1-34) (Teriparatide: Terifrac (rDNA origin) $750 \mathrm{mcg} / 3 \mathrm{ml}$, Intas Pharmaceuticals Ltd, India) (see figure 2).

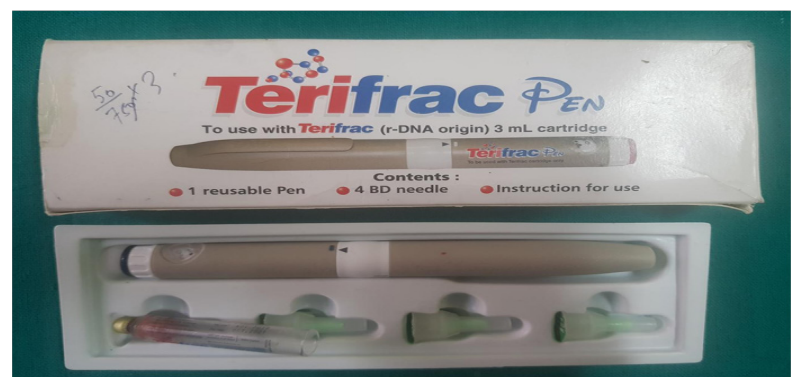

Figure 2: Human recombinant parathyroid hormone analogue 1-34 (Teriparatide: Terifrac ( $r$-DNA origin) $750 \mathrm{mcg} / 3 \mathrm{ml}$, Intas Pharmaceuticals Ltd, India).
Using a reusable pen, teriparatide was instructed to be injected subcutaneously on the lateral aspect of the thigh. $50 \mathrm{mcg}$ or $20 \mathrm{IU}$ of injection was administered every day at the same time. Side effects of headache and pain on the injection site occurred for a few days but was relieved on plain paracetamol tablets. Total calcium and ionized calcium level started to rise. Thereafter, the daily dose of calcium and calcitriol was gradually decreased. Normocalcemia was restored and symptoms completely resolved. On $2020 / 08 / 02$, the patient was discharged on injection Teriparatide $50 \mathrm{mcg}$ (20 IU) s/c once a day, tablet calcium carbonate $3 \mathrm{gm} /$ day, tablet calcitriol $0.25 \mathrm{mcg} /$ day and tablet levothyroxine $150 \mathrm{mcg} /$ day. At the time of discharge, patient had stable vitals and lab parameters were: serum Total calcium 10.1 (8.4-10.2) mg/dL, Ionised calcium 1.01 (1.05-1.27) $\mathrm{mmol} / \mathrm{L}$, phosphorus 3.6 (2.54.5) $\mathrm{mg} / \mathrm{dL}$, magnesium 0.98 (0.66-1.07) $\mathrm{mmol} / \mathrm{L}$, albumin 4.8 (3.5-5) g/dL, and TSH 14.14 (0.3-4.5) $\mathrm{uIU} / \mathrm{mL}$. (see figure 3 ) 


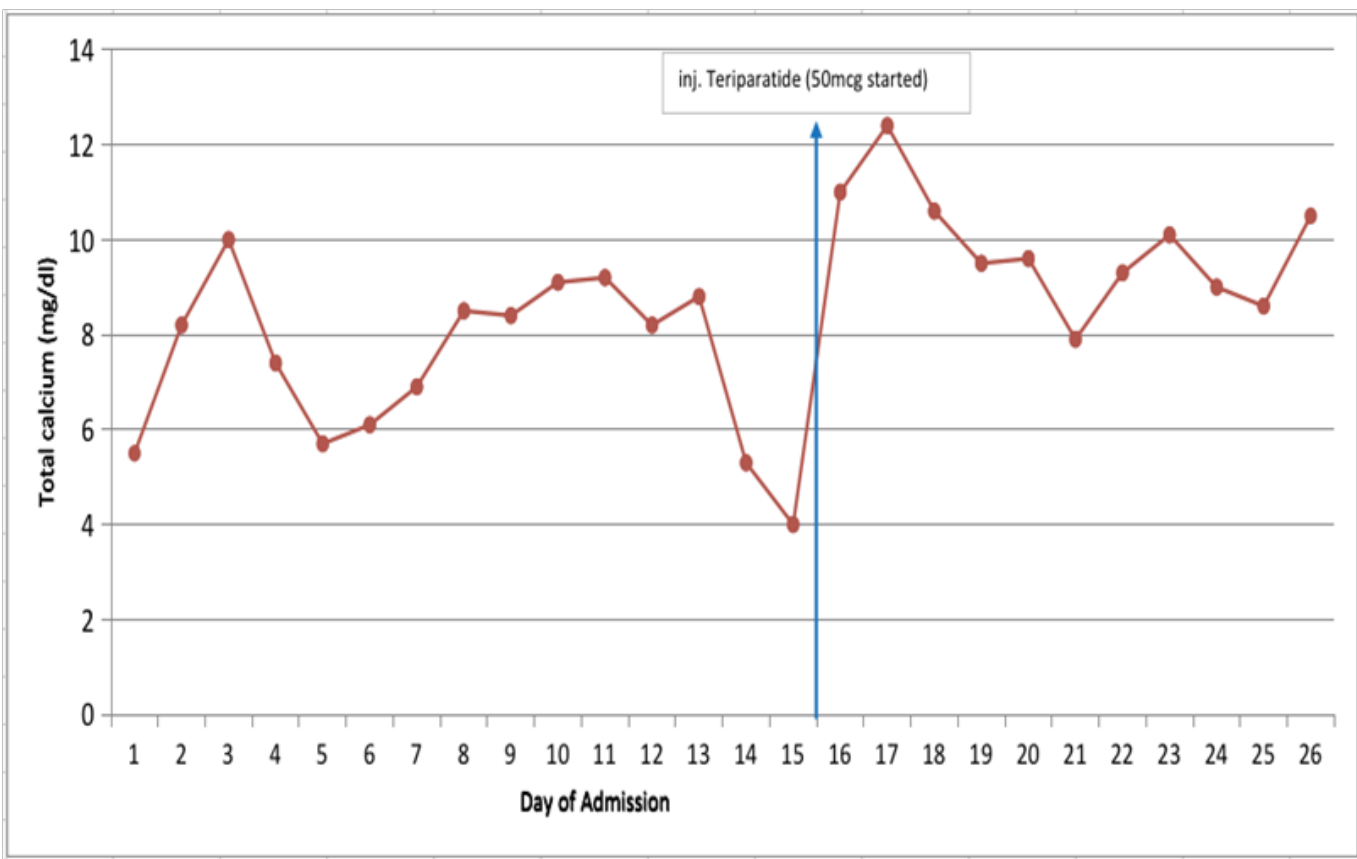

Figure 3: Total calcium measured everyday. Note the high spike in calcium level before initiation of teriparatide is due to intermittent bolus of injection calcium gluconate. After 15 days, injection teriparatide was given as 50 mcg (20 IU) subcutaneous once a day. Calcium infusions were stopped and tablet calcium and vitamin gradually decreased. When needed, serum calcium was adjusted for albumin by the following formula: (0.8 [4.0 - patient's albumin] + serum calcium)

\section{Discussion}

In the majority of patients with hypoparathyroidism secondary to post-total-thyroidectomy, hypocalcemia and its associated symptoms can be treated adequately by calcium and active vitamin D analogs. Hypoparathyroidism which does not respond to this standard management is rare. The success rate of parathyroid transplantation is very low worldwide as most trials have resulted in transplant rejections ${ }^{5}$. Therefore, it is reasonable to use PTH analogs (teriparatide) as hormone replacement therapy in such patients. PTH(1-34), (teriparatide) is absorbed rapidly after subcutaneous injection. It has a half-life of 1 hour, with a bioavailability of $95 \%$. it has been observed that 4-6 hours after subcutaneous injection of teriparatide, a peak increase in serum calcium level by approx. $0.4 \mathrm{mg} / \mathrm{dL}$ occurs, and that it persists for about 6 hours and return to baseline levels after 16-24 hours $^{6}$. Previously, teriparatide was focused more on the treatment of osteoporosis ${ }^{7-8}$. In recent years, after the FDA approval, PTH analogs have been started to be used for indications like iatrogenic hypoparathyroidism? ${ }^{7}$.

For 25 years, Winer et al have led a series of systemic investigations into synthetic human PTH(1-34) replacement in hypoparathyroidism irrespective of any etiology. They first investigated once daily PTH(1-34) in 10 adult hypo-parathyroid subjects in a 20 -week randomized crossover study of daily PTH(1-34) vs calcium and vitamin D supplementation, which showed that PTH(1-34) achieved superior results by maintaining normal serum calcium and urinary calcium excretion levels, although diminishing effects in serum calcium were seen 12 hours after administration 9. This can be explained by the short half-life of PTH (1-34). A 28-week study by the same group comparing once daily with twice-daily injections showed that twice-daily dosing with PTH (1-34) produced significantly higher serum calcium levels 
Use of parathyroid hormone analog (Teriparatide) in patients Jour of Diab and Endo Assoc of Nepal 2020; 4 (2): (29-35) ISSN Print 2594-3367 ISSN Online 2631-2107 with less fluctuation throughout the day. Besides, twice daily injections were superior to oncedaily injection in reducing the urinary calcium level as well as the total daily dose of PTH(1-34) required for stable calcium level ${ }^{10}$. A longer 3-year randomized study by Winer et al showed that twicedaily subcutaneous PTH(1-34) to be superior to oral calcium and calcitriol by maintaining normal urinary calcium excretion along with stable bone density scores ${ }^{11}$. Other studies also showed better serum calcium homeostasis in multiple dosing of teriparatide than single dosing per day. ${ }^{12-13}$

In 2012, a 6 month-randomized crossover trial in chronic postsurgical hypoparathyroidism cases was done. This trial compared continuous PTH(1-34) delivery by a pump with twice-daily delivery of PTH(1-34). In comparison to twice-daily injection, continuous PTH (1-34) delivery by pump resulted to a remarkable decrease in both urine calcium excretion and in the daily $\mathrm{PTH}(1-34)$ dose needed to maintain normal calcium level. Compared to the twice-daily injection regimen, pump delivery was more efficient in normalizing serum calcium and drastically reduced markers of bone turnover. Additionally, pump delivery reduced the excretion of magnesium level and the subsequent need for magnesium supplementation. ${ }^{14}$ In a case of postsurgical hypoparathyroidism, few weeks of teriparatide $20 \mathrm{mcg}$ once daily injection proved sufficient to achieve normocalcemia. However, in severe cases of hypoparathyroidism with associated tubulopathy thrice-daily dosing of teriparatide was needed. ${ }^{13}$ Zafer et al reported that in a patient with treatment-resistant chronic postsurgical hypoparathyroidism, hypocalcemia was not corrected even after teriparatide usage at $60 \mathrm{mcg} /$ day in three divided doses. In this case, intermittent teriparatide infusion using an insulin pump corrected hypocalcemia. ${ }^{15}$ Similar other case reports with postsurgical hypoparathyroidism resistant to standard treatment and subcutaneous injection of teriparatide (once daily or multiple injections) were successfully treated with continuous teriparatide delivery by a pump. ${ }^{16-17}$ In total, teriparatide therapy via pump infusion was found to have less fluctuation in serum calcium, long term stabilization of calcium and phosphate, decreased urinary calcium, and significant reduction of PTH total daily dosage as it mimicked the physiological response of natural endothelial parathyroid hormone found in the human body. Furthermore, teriparatide supplementation in chronic postsurgical hypoparathyroidism decreased "brain fog" like symptoms and improved an individual's mental and physical health ${ }^{18}$.

In our case study, a single subcutaneous daily dose of teriparatide was sufficient to normalize serum calcium levels without needing intravenous calcium infusions. The dose of oral calcium and calcitriol was also gradually reduced. In 10 days of teriparatide administration, we had a fluctuation in serum calcium range from lower limit to higher limit normal range, but never had any episode of hypocalcemia. Further serial calcium level should be measured in the long term follow up to determine the full efficacy of subcutaneous oncedaily dosing of teriparatide. We did not observe any serious adverse reactions besides minor headache and nausea for a few hours, which were attenuated by plain paracetamol and antiemetics. Concerns about carcinogenic potential have been made after the development of osteosarcoma in rats when PTH 1-34 is given for a higher dose and a longer duration $^{19}$. However, multiple studies done on teriparatide over 2 years to 7 years have not shown any carcinogenic risk on humans. ${ }^{20}$

Limitations of this study: Hypocalcemia can be observed in patients with postoperative hypoparathyroidism and an underlying condition can make calcium deficiency even worse. We did not rule out those underlying conditions like impaired calcium absorption from the gastrointestinal tract (D-xylose absorption test, anti-tissue transglutaminase antibody for celiac disease), and tubulopathy associated with excessive urinary calcium loss. 
Use of parathyroid hormone analog (Teriparatide) in patients Jour of Diab and Endo Assoc of Nepal 2020; 4 (2): (29-35) ISSN Print 2594-3367 ISSN Online 2631-2107

\section{Conclusion}

There is a chance of chronic hypoparathyroidism in patients with post total thyroidectomy that would present with recurrent hypocalcemia beyond 6 months, which were not managed with the standard protocol of high dose calcium and active vitamin tablets. PTH hormone analog 1-34 (Teriparatide) represents a new alternative option, with a relatively safe therapeutic profile, providing a long term calcium and phosphate homeostasis, while reducing the need of oral vitamin and calcium supplementation.

\section{References}

1. Reza A, Christian P, Klaus K, Christian S, Bruno N. Hypoparathyroidism after total thyroidectomy: a prospective study. Arch Surg. 2008 Feb;143(2):132-7.

2. Maria LB, John PB, Dolores S, et al. Management of Hypoparathyroidism: Summary statement and guidelines. J Clin Endocrinol Metab. 2016 Jun;101(6):2273-83.

3. Wiebke A, Christian F, Frank C, et al. Wellbeing, mood and calcium homeostasis in patients with hypoparathyroidism receiving standard treatment with calcium and vitamin D. Eur J Endocrinol. 2002 Feb;146(2):215:22.

4. John PB, Maria LB, Natalie EC, et al. Management of Hypoparathyroidism: Present and Future. J Clin Endocrinol Metab. 2016 Jun; 101(6):2313-2324.

5. NM Torregrosa, JM Rodriguez, S Liorente, et al. Definitive treatment for persistent hypoparathyroidism in a kidney transplant patient: parathyroid allotransplantation. Thyroid; 2005; Nov;15(11):1299-302.

6. R Lindsay, J Nieves, E Henneman, V Shen, $\mathrm{F}$ Cosman. Subcutaneous administration of the amino-terminal fragment of human parathyroid hormone-(1-34): kinetics and biochemical response in estrogenized osteoporotic patients. J Clin Endocrinol Metab. 1993 Dec;77(6):1535-9.

7. Marilyn Lee Cheng and Vishal Gupta. Teriparatide-Indications beyond osteoporosis. Indian J Endocrinol Metab; 2012; May-Jun; 16(3):343-348.
8. R Lindsay, JH Krege, F Marin, L Jin, JJ Stepan. Teriparatide for osteoporosis: importance of the full course. Osteoporosis Int; 2006; 27:2395-2410.

9. KK Winer, JA Yanovski, GB Cutler Jr. Synthetic human parathyroid hormone 1-34 vs calcitriol and calcium in the treatment of hypoparathyroidism. JAMA. 1996 Aug 28;276(8):631-6.

10. KK Winer, JA Yanovski, B Sarani, GB Cutler Jr. A randomized, cross-over trial of once-daily versus twice-daily parathyroid hormone 1-34 in treatment of hypoparathyroidism. J Clin Endocrinol Metab. 1998 Oct;83(10):3480-6.

11. Karen KW, Chia WK, James CR, et al. Longterm treatment of hypoparathyroidism: a randomized controlled study comparing parathyroid hormone-(1-34) versus calcitriol and calcium. J Clin Endocrinol Metab. 2003 Sep;88(9):4214-20.

12. KK Winer, Ninet S, Donna P, Bruno S Jr, and Gordon B Cutler Jr. Effects of once versus twice-daily parathyroid hormone 1-34 therapy in children with hypoparathyroidism. J Clin Endocrinol Metab. 2008 Sep;93(9):33893395.

13. Elzbieta AM, Ewa Z, Agnieszka KP, et al. Treatment of severe life threatening hypocalcemia with recombinant human teriparatide in patients with postoperative hypoparathyroidism - a case series. Endokrynol Pol. 2016;67(4):403-12.

14. KK Winer, Bo Zhang, Joseph AS, et al. Synthetic human parathyroid hormone 1-34 replacement therapy: A randomized crossover trial comparing pump versus injections in the treatment of chronic hypoparathyroidism. J Clin Endocrinol Metab. 2012 Feb;97(2):391399.

15. Zafer Pekkolay, Faruk kilinc, Hikmet Soylu, et al. Alternative treatment of resistant hypoparathyroidism by intermittent infusion of teriparatide using an insulin pump: A case report. Turk J Phys Med Rehabil. 2019 Jun;65(2):198-201. 
16. Manel PD, Gonzalo D, Joanna N, Cristian F, Sergio R, Irene H. Successful treatment of vitamin D unresponsive hypoparathyroidism with multipulse subcutaneuos infusion of teriparatide. Eur J Endocrinol. 2008 Nov;159(5):653-7.

17. Carmina Teresa Fuss, Stephanie Burger-Stritt, Silke Horn, et al. Continuous rhPTH (1-34) treatment in chronic hypoparathyroidism. Endocrinol Diabetes Metab Case Rep. 2020 May 29;2020:EDM29-0009.

18. Andrea Palermo, Assunta Santonati, Gaia Tabacco, et al. PTH(1-34) for surgical hypoparathyroidism: A 2-year prospective,
(ㄷ) (1) ( ) P

open-label investigation of efficacy and quality of life. J Clin Endocrinol Metab. 2018 Jan 1;103(1):271-280.

19. John L Vahle, Masahiko Sato, Gerald G Long, et al. Skeletal changes in rats given daily subcutaneous injections of recombinant human parathyroid hormone (1-34) for 2 years and relevance to human safety. Toxicol Pathol; May-Jun 2002;30(3):312-21.

20. Gemma Marcucci, Giuseppe Della Pepa, Maria Luisa Brandi. Drug safety evaluation of parathyroid hormone for hypocalcemia in patients with hypoparathyroidism. Expert Opin Drug Saf; 2017 May;16(5):617-625. 